\title{
Enregistrement et publication des essais cliniques
}

\author{
B. Burnand, M. Rège Walther
}

Correspondance:

Prof. Bernard Burnand CepiC

Institut universitaire de médecine sociale et préventive

Rue du Bugnon 17

CH-1005 Lausanne

Tél. 0213147255

Fax 0213144954

E-mail: Bernard.Burnand@hospvd.ch
Dans l'Ordonnance sur les essais cliniques de produits thérapeutiques, les essais cliniques sont «des études expérimentales réalisées sur l'être humain qui permettent de vérifier de manière systématique la sécurité, l'efficacité et d'autres propriétés d'un produit thérapeutique ou la biodisponibilité» [1]. En Suisse, la réglementation qui traite des essais cliniques est essentiellement centrée sur le médicament et les dispositifs médicaux, alors que l'essai clinique permet théoriquement d'examiner l'efficacité de la plupart des interventions de soins de santé. L'essai clinique randomisé est à l'heure actuelle le meilleur plan d'étude pour examiner l'efficacité d'une intervention médicale ou de soin. Ainsi, parce que les résultats de ces essais sont une source importante de connaissances pour ledéveloppement de la recherche médicale et le transfert de connaissances nouvelles dans la pratique, il est essentiel qu'ils soient portés à la connaissance des professionnels de la santé et des patients.

Des milliers d'essais cliniques sont menés chaque année. Certains d'entre eux se déroulent sans que personne ne soit au courant de leur existence en dehors de leurs promoteurs, et leurs résultats ne sont jamais publiés. Dans d'autres cas, seule une partie des résultats obtenus est publiée.

La décision de ne pas publier les résultats d'un essai clinique et l'omission sélective de certains résultats ont des conséquences qui peuvent être potentiellement fatales. Une illustration récente se rapporte au traitement de la dépression chez les enfants et les adolescents par des inhibiteurs sélectifs de la recapture de la sérotonine (ISRS).

Une revue systématique publiée dans Lancet [2] a comparé les résultats des études publiées et non publiées rapportant les inconvénients et les bénéfices de ces médicaments. Il est apparu que les risques liés au traitement par certains de ces antidépresseurs étaient largement plus élevés que les bénéfices. Le poids des résultats non publiés a été significatif dans la démonstration puisque selon les études publiées, les bénéfices étaient supérieurs aux risques (tendances suicidaires plus fréquent lors du traitement avec des ISRS).
Cette étude démontre ainsi clairement que la non-publication de résultats d'essais pour quelque raison que ce soit, ou l'omission de rapporter des effets importants dans les essais publiés, peut conduire à des recommandations de traitement erronées.

Cette non-publication de résultats est également indéfendable d'un point de vue éthique. En effet, les patients qui consentent à participer à un processus d'essai clinique en acceptant les risques qui lui sont liés et en sachant que souvent, ils n'en retireront pas un bénéfice direct, attendent au moins qu'il en résulte des éléments utiles pour faire avancer les recherches dans le domaine des soins, au bénéfice de toutes les personnes qui en auraient besoin. Ne pas publier les résultats de ces essais revient ainsi à tromper les participants.

L'une des solutions actuellement proposée pour éviter de tels problèmes est la mise en place d'un registre mondial centralisé d'essais cliniques, indépendant et d'accès public [3, 4]. Il aurait l'avantage d'obliger les promoteurs des essais cliniques à assumer leur responsabilité éthique, scientifique et économique [5]. Un tel registre permettrait d'identifier et de prévenir les biais dus à la publication incomplète ou à la nonpublication de résultats, d'identifier les essais à mener pour compléter les études déjà effectuées, permettant ainsi de mieux planifier la recherche clinique, de décider des priorités et d'éviter des duplications.

Ainsi, tout essai clinique passé, prévu ou en cours devrait être obligatoirement inscrit dans ce registre mondial, avec un identifiant unique délivré par une organisation reconnue internationalement. Les protocoles et les caractéristiques-clés de l'essai devraient y figurer également. Les principaux résultats de l'essai devraient également être obligatoirement publiés.

A terme, l'enregistrement devrait également être exigé pour d'autres types d'études susceptibles de fournir des résultats importants quant à l'efficacité ou aux risques des interventions de soins, comme par exemple des études de surveillance des effets indésirables après mise sur le marché. 
Un tel registre centralisé n'a pas encore vu le jour. Il en existe dans différentes régions du monde mais ils sont limités par leur couverture géographique et/ou par les aspects médicaux auxquels ils se réfèrent. Aucun d'eux ne fonctionne sur une base obligatoire, définie dans une loi. Chacun défini sa propre politique, avec ses critères et sans vue d'ensemble au niveau mondial. De plus, pour certains d'entre eux, l'accès public est limité.

Parmi les registres existants, on peut citer le registre mis en place par le United States National Institutes of Health [6] au travers de la National Library of Medicine. La Fondation Européenne de la Science [7] a publié des directives recommandant l'enregistrement des essais cliniques et suggérant fortement à ses membres de mettre sur pied un registre. De son côté, l'Organisation Mondiale de la Santé (OMS) [8] a décidé que tous les essais cliniques randomisés approuvés par sa commission d'éthique se verraient assigner un identifiant (International Standard Randomised Controlled Trial Number) [9] qui augmentera la visibilité et l'accessibilité des essais soutenus par l'OMS.

En Suisse, Swissmedic, l'autorité subordonnée au Département fédéral de l'intérieur, chargée de l'autorisation de mise sur le marché, du contrôle de la fabrication et de la qualité ainsi que de la surveillance du marché des produits thérapeutiques, possède un registre des essais cliniques conduits avec des médicaments. Ce dernier n'est cependant pas accessible au public.

Le tableau éclaté présenté ci-dessus semble devoir peu à peu se modifier: des voix s'élèvent un peu partout pour exiger une plus grande transparence et une meilleure coordination. Une initiative majeure a été prise récemment par le Comité international des éditeurs de journaux médicaux (ICMJE) qui a décidé de ne plus publier les résultats d'essais cliniques qui ne seraient pas enregistrés dès leur démarrage dans un registre public [10]. Ce comité veut obliger les auteurs à publier les résultats des essais qu'ils conduisent, y compris les résultats négatifs.

En Suisse, un prochain changement majeur en direction d'une plus grande transparence consisterait à modifier les bases légales par le biais notamment de la future Loi fédérale sur la recherche impliquant des êtres humains, afin que l'accès au registre des essais cliniques en cours puisse être public et inclure tous les essais évaluant des interventions médicales ou de soins ayant un effet potentiel chez l'homme. A terme, il est souhaitable que le registre suisse puisse faire partie d'une organisation plus large, européenne, voir mondiale!

\section{Références}

1 Ordonnance sur les essais cliniques de produits thérapeutiques (Oclin). RS 812.214.2, sec. 1, art. 5.

2 Whittington CJ, Kendall T, Fonagy P, Cottrell D, Cotgrove A, Boddington E. Selective serotonine reuptake inhibitors in childhood depression: systematic review of published versus unpublished data. Lancet 2004;363:1341-5.

3 Steinbrook R. Public registration of clinical trials. N Engl J Med 2004;351:315-7.

4 www.cochrane.org/news/articles/2004.07.26.htm

5 Antes G. Registering clinical trials is necessary for ethical, scientific and economic reasons. Bulletin of the World Health Organisation 2004;82:321.

6 McCray AT. Better access to information about clinical trials. Ann Intern Med 2002;133:609-14.

7 Controlled clinical trials. European Science Foundation Policy Briefing 13. Strasbourg: European Science Foundation; 2001. www.esf.org/ publication/90/espb13.pdf.

8 World Health Organization. WHO leads drive for internal coordination of clinical research. Press release $\mathrm{WHO} / 23,2$ April 2004.

9 www.controlled-trials.com.

10 International Committee of Medical Journal Editors (ICMJE). Uniform Requirements for Manuscripts Submitted to Biomedical Journals: Writing and Editing for Biomedical Publication. Updated October 2004. www.icmje.org. 